January 24, 2012

Ms. Laurie Kauffman

U.S. Nuclear Regulatory Commission

Division of Nuclear Materials Safety

475 Allendale Road

King of Prussia, PA 19406-1415

\title{
SUBJECT: LETTER REPORT - INDEPENDENT CONFIRMATORY SURVEY RESULTS OF SOILS ASSOCIATED WITH THE ARGYLE STREET SEWER LINE AT THE UNITED NUCLEAR CORPORATION NAVAL PRODUCTS SITE, NEW HAVEN, CONNECTICUT DCN: 2040-SR-01-0
}

Dear Ms. Kauffman:

Oak Ridge Institute for Science and Education (ORISE) personnel visited the United Nuclear Corporation (UNC) Naval Products site on three separate occasions during the months of October and November 2011. The purpose of these visits was to conduct confirmatory surveys of soils associated with the Argyle Street sewer line that was being removed.

Soil samples were collected from six different, judgmentally determined locations in the Argyle Street sewer trench. In addition to the six soil samples collected by ORISE, four replicate soil samples were collected by Cabrera Services, Inc. (CSI) for analysis by the ORISE laboratory. Replicate samples S0010 and S0011 were final status survey (FSS) bias samples; S0012 was an FSS systematic sample; and S0015 was a waste characterization sample. Six soil samples were also collected for background determination. Uranium-235 and uranium-238 concentrations were determined via gamma spectroscopy; the spectra were also reviewed for other identifiable photopeaks. Radionuclide concentrations for these soil samples are provided in Table 1.

Total uranium concentrations for the soil samples ranged from 0.70 to 22 picocuries per gram $(\mathrm{pCi} / \mathrm{g})$. The two soil samples with the highest concentrations of total uranium were collected from inside the sewer pipe. The pipe has since been removed. The remaining soil samples were below $3 \mathrm{pCi} / \mathrm{g}$. All soil samples analyzed by the ORISE laboratory were below the derived concentration guideline level $\left(\mathrm{DCGL}_{\mathrm{w}}\right)$ of $30 \mathrm{pCi} / \mathrm{g}$ for total uranium.

In addition to the replicate samples and the samples collected by ORISE, CSI submitted three soil samples for inter-laboratory ${ }^{*}$ comparison analyses (Table 2). One sample was from the background reference area, one was from waste characterization efforts (material inside the sewer line), and one was a FSS sample. The inter-laboratory comparison analyses results between ORISE and CSI were in agreement, except for one sample collected in the reference area. Additional information will be requested by ORISE to explain the difference and will be presented in the final report. Additionally, since one of the samples analyzed was close to the DCGL w $_{\mathrm{w}}$ and the results between ORISE and CSI were in agreement, ORISE is confident that CSI is able to accurately determine whether a given

\footnotetext{
*While the term "laboratory" is used, CSI analyzes soil samples with a portable gamma spectroscopy system.
} 
sample is below the release criteria.

Once the Argyle Street sewer entered Shelton Avenue, the pipe did not follow the path as anticipated, therefore CSI decided to characterize the pipe and, if the analytical results met the release guidelines, abandon it in place. CSI personnel also collected sludge material from inside the pipe (samples mentioned previously), and smears were also collected at one foot intervals inside the pipe (at the 3, 6, 9 and 12 o'clock positions at each one foot interval); the distance inside the pipe was limited to what was easily accessible from the pipe openings at either end (approximately three feet). CSI personnel collected replicate smears for ORISE. Gross alpha activity ranged from 0 to 49 disintegrations per minute per one hundred square centimeters $\left(\mathrm{dpm} / 100 \mathrm{~cm}^{2}\right)$ and gross beta activity ranged from -5 to $19 \mathrm{dpm} / 100 \mathrm{~cm}^{2}$. The complete results for the smears are provided in Table 3; ORISE has requested CSI's smear analysis and will make a comparison in the final report.

A full confirmatory survey report will follow after completion of all confirmatory survey activities. Please contact me at 865.574.6273, or Wade Adams at 865.576.0065, should you have any questions.

Sincerely,

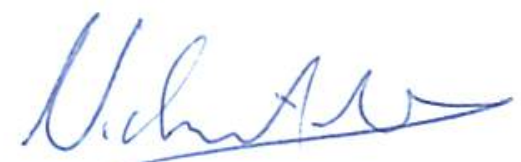

Nick A. Altic

Health Physicist/Assistant Project Manager

Independent Environmental Assessment

and Verification

NAA:fr

Enclosure

c:

T. Carter, NRC/FSME/DWMEP/DD/SPT-85

S. Nesmith, NRC/TAPM/TWFN 8A23

Electronic distribution:
S. Roberts/ORISE
E. Bailey/ORISE
T. Vitkus/ORISE
W. Adams/ORISE

File/2040

\begin{tabular}{|l|l|}
\hline Distribution approval and concurrence: & Initials \\
\hline Technical Review & \\
\hline Laboratory Review & \\
\hline Quality Review & \\
\hline
\end{tabular}




\begin{tabular}{|c|c|c|c|c|c|c|c|c|c|c|c|}
\hline \multicolumn{12}{|c|}{$\begin{array}{l}\text { Table 1. Radionuclide Concentrations in Soil Samples } \\
\text { UNC Naval Products, New Haven, Connecticut }\end{array}$} \\
\hline \multirow{2}{*}{ Sample ID } & \multicolumn{2}{|c|}{ Gamma Count Rate (cpm) } & \multicolumn{9}{|c|}{ Radionuclide Concentrations (pCi/g) } \\
\hline & Pre-sample & Post-sample & & $\mathrm{J}-23$ & & & $\mathrm{U}-2$ & & & tal & \\
\hline \multicolumn{12}{|c|}{ Reference Area Background Samples } \\
\hline S0001 & 7,429 & 8,447 & 0.14 & \pm & $0.11^{\mathrm{b}}$ & 1.22 & \pm & 0.27 & 2.58 & \pm & 0.55 \\
\hline S0002 & 6,681 & 7,160 & 0.08 & \pm & 0.12 & 0.86 & \pm & 0.29 & 1.80 & \pm & 0.59 \\
\hline S0003 & 6,874 & 8,067 & -0.04 & \pm & 0.13 & 0.56 & \pm & 0.20 & 1.08 & \pm & 0.42 \\
\hline S0004 & 6,540 & 7,651 & 0.03 & \pm & 0.11 & 0.40 & \pm & 0.23 & 0.83 & \pm & 0.47 \\
\hline S0005 & 7.639 & 8,375 & -0.12 & \pm & 0.13 & 0.47 & \pm & 0.28 & 0.82 & \pm & 0.57 \\
\hline S0006 & 6,175 & 6,560 & 0.21 & \pm & 0.12 & 0.66 & \pm & 0.21 & 1.53 & \pm & 0.44 \\
\hline \multicolumn{12}{|c|}{ Argyle Street Sewer Line Samples } \\
\hline S0007 & 9,786 & 11,194 & $0.00^{c}$ & \pm & 0.11 & 0.40 & \pm & 0.25 & 0.80 & \pm & 0.51 \\
\hline S0008 & 9,244 & 9,774 & 0.06 & \pm & 0.11 & 0.57 & \pm & 0.17 & 1.20 & \pm & 0.36 \\
\hline S0009 & 7,274 & 8,430 & 0.03 & \pm & 0.09 & 0.54 & \pm & 0.38 & 1.11 & \pm & 0.77 \\
\hline S0013 & 4,259 & 4,536 & 0.04 & \pm & 0.12 & 0.40 & \pm & 0.33 & 0.84 & \pm & 0.67 \\
\hline S0014 & 4,250 & 4,309 & 0.04 & \pm & 0.12 & 0.55 & \pm & 0.21 & 1.14 & \pm & 0.44 \\
\hline S0016 & 4,312 & 4,788 & 0.01 & \pm & 0.11 & 0.43 & \pm & 0.30 & 0.87 & \pm & 0.61 \\
\hline \multicolumn{12}{|c|}{ Arglye Street Replicate Samples (Collected by CSI Personnel) } \\
\hline S0010 & -- & -- & 0.04 & \pm & 0.12 & 0.33 & \pm & 0.04 & 0.70 & \pm & 0.14 \\
\hline S0011 & -- & -- & 0.08 & \pm & 0.13 & 0.40 & \pm & 0.18 & 0.88 & \pm & 0.38 \\
\hline S0012 & -- & -- & 0.00 & \pm & 0.10 & 0.79 & \pm & 0.43 & 1.58 & \pm & 0.87 \\
\hline S0015d & -- & -- & 0.78 & \pm & 0.10 & 0.61 & \pm & 0.25 & 22 & \pm & 3 \\
\hline \multicolumn{12}{|c|}{ Inter-laboratory Comparison Analyses Samples } \\
\hline S0017d & -- & -- & 0.69 & \pm & 0.11 & 0.55 & \pm & 0.30 & 20 & \pm & 3 \\
\hline S0018 & -- & -- & 0.08 & \pm & 0.07 & 0.72 & \pm & 0.39 & 1.52 & \pm & 0.78 \\
\hline S0019 & -- & -- & -0.01 & \pm & 0.12 & 0.24 & \pm & 0.21 & 0.47 & \pm & 0.44 \\
\hline
\end{tabular}

${ }^{a}$ Total U was calculated as U-235 + (2*U-238) for natural U and U-238 + (28*U-235) for enriched uranium samples. bUncertainties represent the $95 \%$ confidence level, based on total propagated uncertainties.

'Zero values are due to rounding.

dSamples contained enriched uranium. 


\begin{tabular}{|c|c|c|c|c|c|c|c|c|}
\hline \multicolumn{9}{|c|}{$\begin{array}{l}\text { Table 2. Inter-Laboratory Comparison Analyses } \\
\text { UNC Naval Products, New Haven, Connecticut }\end{array}$} \\
\hline \multirow{2}{*}{ ORISE Sample ID } & \multirow{2}{*}{ CSI Sample ID } & \multicolumn{7}{|c|}{ Total U Reported (pCi/g) } \\
\hline & & \multicolumn{3}{|c|}{ ORISE } & \multicolumn{3}{|c|}{ CSI } & DER $^{\mathrm{a}}$ \\
\hline S0017 & UNC-WCJ-SR-012 & 20 & \pm & 3 & 21.80 & \pm & 0.27 & 0.6 \\
\hline S0018 & UNC-FSS-RA-012 & 1.52 & \pm & 0.78 & 4.05 & \pm & 0.07 & 3.2 \\
\hline S0019 & UNC-FSS-SR-037B & 0.47 & \pm & 0.44 & 2.15 & \pm & 2.24 & 0.7 \\
\hline
\end{tabular}

${ }^{a}$ Absolute Relative Difference (DER) was calculated based on the formula in the Department of Energy Quality Systems for Analytical Services, Rev 2.7, and provides a way to determine if analytical results of duplicates are in agreement. A DER $\leq 3$ means the duplicates are in agreement, 3 represents the $99 \%$ confidence level. 


\begin{tabular}{|c|c|c|c|c|c|}
\hline & $\begin{array}{l}\text { able } 3 . \mathrm{Sn} \\
\text { UNC Na }\end{array}$ & $\begin{array}{l}\text { esults For } f \\
\text { oducts, } \mathrm{Ne}\end{array}$ & $\begin{array}{l}\text { e Street Sev } \\
\text { aven, Conn }\end{array}$ & $\begin{array}{l}\text { Pipes } \\
\text { cut }\end{array}$ & \\
\hline \multirow[t]{2}{*}{ End of Pipe } & \multirow{2}{*}{$\begin{array}{c}\text { Interval } \\
(\mathrm{ft})\end{array}$} & \multirow{2}{*}{$\begin{array}{l}\text { Location } \\
\text { (o'clock) }\end{array}$} & \multirow[t]{2}{*}{ Smear ID } & \multicolumn{2}{|c|}{$\begin{array}{l}\text { Smear Activity } \\
\left(\mathrm{dpm} / 100 \mathrm{~cm}^{2}\right)\end{array}$} \\
\hline & & & & Alpha & Beta \\
\hline \multirow{16}{*}{ West } & \multirow{4}{*}{$0-1$} & 6 & R0001 & 2 & 7 \\
\hline & & 9 & R0002 & 0 & 3 \\
\hline & & 12 & R0003 & 49 & -1 \\
\hline & & 3 & R0004 & 0 & -5 \\
\hline & & 6 & R0005 & 0 & 1 \\
\hline & & 9 & R0006 & 2 & 1 \\
\hline & $1-2$ & 12 & R0007 & 2 & -4 \\
\hline & & 3 & R0008 & 0 & -1 \\
\hline & & 6 & R0009 & 0 & -1 \\
\hline & 23 & 9 & R0010 & 0 & -4 \\
\hline & $2-3$ & 12 & R0011 & 0 & -2 \\
\hline & & 3 & R0012 & 0 & -1 \\
\hline & & 6 & R0013 & 6 & 3 \\
\hline & & 9 & R0014 & 4 & -4 \\
\hline & $3-4$ & 12 & R0015 & 0 & -1 \\
\hline & & 3 & R0016 & 0 & -2 \\
\hline & & 6 & R0017 & 6 & -2 \\
\hline & $0-1$ & 12 & R0018 & 0 & -3 \\
\hline Eop & 12 & 6 & R0019 & 28 & 5 \\
\hline East & $1-2$ & 12 & R0020 & 0 & -2 \\
\hline & & 6 & R0021 & 30 & 19 \\
\hline & $2-3$ & 12 & R0022 & 0 & -1 \\
\hline
\end{tabular}

